\title{
Radiation-induced matrix metalloproteinases limit natural killer cell-mediated anticancer immunity in NCI-H23 lung cancer cells
}

\author{
WOONG HEO ${ }^{1}$, YOUNG SHIN LEE ${ }^{1}$, CHEOL HUN SON ${ }^{1,2}$, KWANGMO YANG ${ }^{2}$, \\ YOU SOO PARK ${ }^{2}$ and JAEHO BAE ${ }^{1}$ \\ ${ }^{1}$ Department of Biochemistry, Pusan National University School of Medicine, Yangsan, Gyeongsangnam-do 626-770; \\ ${ }^{2}$ Department of Research Center, Dongnam Institute of Radiological and Medical Sciences, \\ Busan, Gijang 619-953, Republic of Korea
}

Received April 30, 2014; Accepted August 12, 2014

DOI: $10.3892 / \mathrm{mmr} .2014 .2918$

\begin{abstract}
Radiotherapy has been used to treat cancer for $>100$ years and is required by numerous patients with cancer. Ionizing radiation effectively inhibits the growth of cancer cells by inducing cell death and increasing anticancer immunity, through the induction of natural killer group 2 membe r D ligands (NKG2DLs); however, adverse effects have also been reported, including the promotion of metastasis. Matrix metalloproteinases (MMPs) are induced by ionizing radiation and have an important role in the invasion and metastasis of cancer cells. Previously, MMPs were demonstrated to increase the shedding of NKG2DLs, which may reduce the surface expression of NKG2DLs on cancer cells. As a consequence, the cancer cells may escape natural killer (NK)-mediated anticancer immunity. In the present study, NCI-H23 human non-small cell lung cancer cells were used to investigate the combined effects of ionizing radiation and MMP inhibitors on the expression levels of NKG2DLs. Ionizing radiation increased the expression of MMP2 and ADAM metalloproteinase domain 10 protease, as well as NKG2DLs. The combined treatment of ionizing radiation and MMP inhibitors increased the surface expression levels of NKG2DLs and resulted in the increased susceptibility of the cancer cells to NK-92 natural killer cells. Furthermore, soluble NKG2DLs were increased in the media by ionizing radiation and blocked by MMP inhibitors. The present study suggests that radiotherapy may result in the shedding of soluble NKG2DLs, through the induction
\end{abstract}

Correspondence to: Professor Jaeho Bae, Department of Biochemistry, School of Medicine, Pusan National University, 707 Busandaehakro-49, Beomeo-ri, Mulgeum-eup, Yangsan-si, Gyeongsangnam-do 626-770, Republic of Korea

E-mail: biosole@pusan.ac.kr

Key words: natural killer group 2 member D ligands, ionizing radiation, matrix metalloproteases of MMP2, and combined treatment with MMP inhibitors may minimize the adverse effects of radiotherapy.

\section{Introduction}

Ionizing radiation breaks DNA double strands and results in the induction of various responses in cancer cells, including altered susceptibility to immune cells. It achieves this through the upregulation of immunomodulatory surface and secretory molecules, such as the major histocompatibility complex (MHC), co-stimulatory molecules and cytokines (1). It has previously been shown that irradiation of malignant cells enhanced the natural killer (NK) cell-mediated cytotoxicity in autologous models (2) and the susceptibility of cancer cells to NK cells has been shown to increase following irradiation (3). However, despite the beneficial effects of radiotherapy in the treatment of cancer, there are some adverse effects, including facilitating invasion and metastasis $(4,5)$. It has previously been observed that matrix metalloproteinases (MMPs) are upregulated by irradiation and their expression has been associated with poor prognosis of patients with cancer (6-8). Furthermore, it has been shown that several proteases, including MMPs and a disintegrin and metalloproteinase domain-containing proteins (ADAMs), may increase the soluble form of NK group 2 member D ligands (NKG2DLs) (9-13). This may result in cancer cell evasion of NK cell-mediated immune responses, through the reduction of surface NKG2DLs on cancer cells and the downregulation of NKG2D receptors on NK cells (11). Therefore, it may be beneficial to reduce the activity of MMPs and decrease the expression levels of soluble NKG2DLs in cancer radiotherapy. In the present study, MMP activity was inhibited using GM6001 or MMP inhibitor III (MMPi III), and the alterations to the surface and soluble NKG2DLs expression levels were investigated in lung cancer cells, following irradiation.

\section{Materials and methods}

Cell lines and reagents. NCI-H23, human non-small cell lung cancer cells, were obtained from the Korean Cell Line 
Bank (Seoul, South Korea). The cells were maintained in RPMI-1640 media, supplemented with $10 \%$ fetal bovine serum (FBS; Gibco Life Technologies, Carlsbad, CA, USA), 2 mM L-glutamine (Life Technologies, Carlsbad, CA, USA), $100 \mathrm{mg} / \mathrm{ml}$ streptomycin (USB Corporation, Cleveland, $\mathrm{OH}$, USA), and $100 \mathrm{U} / \mathrm{ml}$ penicillin (USB corporation). The NK-92 natural killer cell line was obtained from the American Type Culture Collection (Manassas, VA, USA) and maintained in $\alpha$-Minimum Essential Modified medium, supplemented with $12.5 \%$ (v/v) FBS, $12.5 \%$ (v/v) horse serum (Life Technologies), $2 \mathrm{mM}$ L-glutamine, $0.1 \mathrm{mM}$ 2-mercaptoethanol (Sigma-Aldrich, St. Louis, MO, USA), 200 U/ml recombinant human interleukin-2 (Proleukin; Novartis Pharmaceuticals, Camberley, UK), $100 \mathrm{mg} / \mathrm{ml}$ streptomycin, and $100 \mathrm{U} / \mathrm{ml}$ penicillin. All of the cells were cultured at $37^{\circ} \mathrm{C}$, in a humidified atmosphere containing $5 \% \mathrm{CO}_{2}$.

Total RNA extraction and semi-quantitative polymerase chain reaction ( $q P C R$ ). Total RNA extraction and qPCR were performed as described by previous methods (14). Briefly, total RNA was extracted from the cells using the RNeasy ${ }^{\circledR}$ Mini kit (Qiagen, Hilden, Germany), according to the manufacturer's instructions. cDNA was synthesized from $1 \mu \mathrm{g}$ extracted total RNA, using 100 pmol random primers (Takara Bio Inc., Otsu, Japan) and $100 \mathrm{U}$ M-MLV reverse transcriptase (Promega Corporation, Madison, WI,USA). The resulting cDNA was used in the PCR reaction, which was performed using the QIAGEN Multiplex PCR kit (Qiagen). Numerous primer pairs were used to investigate the mRNA expression levels of NKG2DLs: MHC class-I polypeptide-related chain proteins (MIC)A and MICB, UL-16 binding proteins (ULBP)1-3; MMPS: MMP2, 9 and 14; and ADAMs: ADAM10 and 17 (Bioneer Corporation, Daejeon, Korea). $\beta$-actin and ribosomal protein L19 were used as the loading control and degradation marker, respectively. The PCR products were separated by electrophoresis on an ethidium bromide-stained $2.0 \%$ agarose gel and quantified using Quantity One Image Analysis Software (Bio-Rad Laboratories, Hercules, CA, USA).

Flow cytometry. To determine the surface NKG2D ligands on cancer cells, the cells were incubated with $10 \mathrm{mg} / \mathrm{ml}$ mouse anti-MICA, anti-MICB and anti-ULBP1-3 (R\&D Systems, Inc., Minneapolis, MN, USA), which are NKG2DL-specific monoclonal antibodies (mAbs), or the corresponding isotype controls for $10 \mathrm{~min}$ at room temperature. Subsequently, the cells were incubated with a goat anti-mouse-phycoerythrin conjugated antibody (BD Phamingen, San Diego, CA, USA) for $30 \mathrm{~min}$ at $4{ }^{\circ} \mathrm{C}$. The analysis was performed using a BDFACSCalliber $^{\mathrm{TM}}$ (Becton Dickinson, Franklin Lakes, NJ, USA) using CellQuest ${ }^{\mathrm{TM}}$ software (Becton Dickinson). The cell surface expression was quantified as the value of the mean fluorescence intensities obtained with the specific mAbs.

Irradiation and co-treatment with MMP inhibitors. To irradiate the cancer cells, a ClinaciX Linear Accelerator (Varian Medical Systems, Palo Alto, CA, USA) was used, under the guidance of Dr. Jiho Nam (Pusan National University Yangsan Hospital, South Korea). The $1 * 106$ cells were seeded at prior day to $100 \mathrm{~mm}$ diameter culture dishes with $10 \mathrm{ml}$ PRMI-1640 medium and irradiated at a rate of $8 \mathrm{~Gy} / \mathrm{min}$ to $200 * 200 \mathrm{~mm}$ area, under a $10 \mathrm{~mm}$ depth-coverage of RPMI-1640 medium. The irradiated NCI-H23 cells were allowed to recover for $6 \mathrm{~h}$ after reduction of the medium to $10 \mathrm{ml}$. Subsequently, MMPi III and GM6001 were treated for $18 \mathrm{~h}$. Following an incubation for $18 \mathrm{~h}$, the cells and supernatants were harvested for further experimentation.

NK cell-mediated cytotoxicity assay. NK cell-mediated cytotoxicity was determined using flow cytometry. Briefly, the irradiated only or irradiation/MMP inhibitor co-treated NCI-H23 cells were harvested. The cells were stained with $50 \mathrm{mM}$ carboxyfluorescein succinimidyl ester (CFSE) for $30 \mathrm{~min}$ at $37^{\circ} \mathrm{C}$ and washed three times with phosphate buffered saline (Gibco Life Technologies, Carlsbad, CA, USA). NK-92 cells and CFSE-stained NCI-H23 cells were co-cultured for $4 \mathrm{~h}$. Propidium iodide (PI) was added to the co-cultured samples for identification of the dead cells. The dead cells were quantified using the following formula: $(\mathrm{CFSE}+\mathrm{PI}+$ cells/CFSE + cells x 100).

ELISA. NCI-H23 cells were treated with MMP inhibitors following irradiation, and the media was harvested. The soluble MICA in the media was evaluated using a Human ELISA kit (Abcam, Cambridge, United Kingdom), according to the manufacturer's instructions. Briefly, standard MICA and $100 \mathrm{ml}$ supernatants, which were prepared according to the manufacturer's instructions, were added to anti-MICA coated microplates. Following a $24 \mathrm{~h}$ incubation at $4^{\circ} \mathrm{C}$, biotinylated-MICA detection antibody and horseradish peroxidase-streptavidin solution were sequentially added. After the addition of the substrate reagent, soluble MICA was detected using microplate reader fluorometry at a wavelength of $450 \mathrm{~nm}$ (MicroQuant; MTX Lab Systems, Inc., Vienna, VA, USA).

Statistical analyses. To evaluate the altered gene expression levels, the mean fold of gene expression among the groups and the standard error of the mean were calculated. For comparisons of the groups, a paired Student's t-test was performed. $\mathrm{P}<0.05$ was considered to indicate a statistically significant difference in all of the experiments.

\section{Results}

Ionizing radiation increases the expression levels of MMP2 and ADAM 10, in a dose-dependent manner. Previous reports have shown that three MMPs (MMP2, 9 and 14) and two ADAMs (ADAM10 and 17) are associated with the shedding of MICA and MICB (9-13). Therefore, in the present study, the alterations to the expression levels of these proteases, following ionizing radiation, were analyzed by qPCR. NCI-H23 cells were irradiated using a ClinaciX Linear Accelerator (Varian Medical Systems, Inc.) at 8, 16 and 24 Gy doses. Following a $24 \mathrm{~h}$ incubation of the cells, the mRNA expression levels of three MMPs (MMP2, 9 and 14) and two ADAMs (ADAM10 and 17) were analyzed. MMP2 and ADAM10 were markedly upregulated by ionizing radiation, in a dose-dependent manner (Fig. 1). The expression levels of MMP9 and MMP14 were low and the expression levels of ADAM17 were high, and the alterations to these proteases, in response to irradiation of the cells are not shown. 
Table I. Primer sequences used in multiplex reverse-transcription polymerase chain reaction.

\begin{tabular}{lll}
\hline Name & Polarity & Sequence $\left(5^{\prime}-3^{\prime}\right)$ \\
\hline MMP2 & Forward & ACGGACTCCTGGCTCATGCC \\
MMP9 & Reverse & CTGTCCTTCAGCGTTGCCGC \\
& Forward & CGACCCGAGCTGACTCGACG \\
MMP14 & Reverse & GCGGTGTGGTGGTGGTTGGA \\
ADAM10 & Forward & CCGGTGGTCTCGGACCATGT \\
ADAM17 & Reverse & TGTGGCACTCTCCCACACGC \\
& Forward & TGGAACAGAGTGCACACCAGGA \\
& Reverse & TCTTCCCTCTGGTTGATTTGCATCG \\
& Forward & TGTGCCCCGAATGAGGACCAG \\
\hline
\end{tabular}
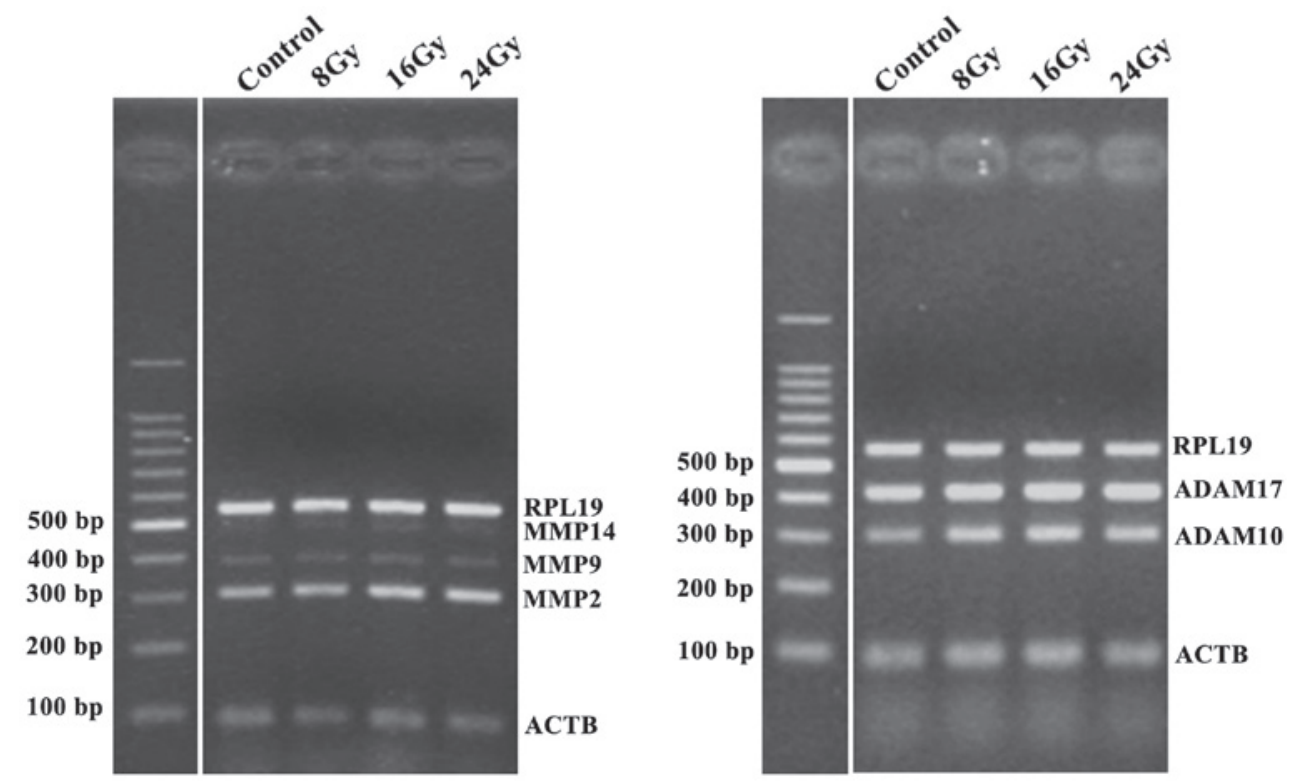

Figure 1. Upregulation of metalloproteinase mRNA expression by ionizing radiation. NCI-H23 non-small cell lung cancer cells were irradiated with the indicated doses $(0,8,16,24 \mathrm{~Gy})$. Following a $24 \mathrm{~h}$ recovery, the cells were harvested and analyzed using quantitative polymerase chain reaction. Ribosomal protein L19 (RPL19) and $\beta$-actin (ACTB) were used as the RNA degradation marker and loading control, respectively. Bp, base pairs; MMP, matrix metalloproteinases; ADAM, a disintegrin and metalloproteinase domain-containing protein; Gy, gray unit.

Surface NKG2DL levels are increased by ionizing radiation, in a dose-independent manner. The surface expression of NKG2DLs in NCI-H23 cells was analyzed using flow cytometric analysis. The surface expression levels of NKG2DLs were markedly increased following 8 and 16 Gy irradiation, in a dose-independent manner (Fig. 2). An irradiation dose of 16 Gy was no more effective at inducing NKG2DLs surface expression, as compared with a $8 \mathrm{~Gy}$ irradiation. These results suggest that ionizing radiation may be a potent inducer of NKG2DLs and that high dose irradiation may have adverse effects, especially in the induction of MICA, MICB and ULBP3. Previous reports have suggested that this upregulation is associated with the ATM-ATR pathway at the translational level $(15,16)$.

MMP inhibitors do not increase the $m R N A$ expression levels of NKG2DLs, but they do increase the levels of surface NKG2DLs. To determine whether the inhibition of MMPs modulated the expression of NKG2DLs, two MMP inhibitors, MMPi III and GM6001, were used to treat the NCI-H23 cells. At the mRNA level, the expression levels of NKG2DLs were not markedly increased, in response to MMP inhibition (Fig. 3A). However, the surface protein levels of MICA and MICB were increased in the cells treated with MMP inhibitors. The surface MICA levels were markedly increased in response to treatment with both of the MMP inhibitors, whereas the surface MICB levels were only slightly increased (Fig. 3B).

Combined treatment of ionizing radiation and MMP inhibitors increases surface MICA levels. To determine whether the inhibition of MMPs, which were induced by ionizing radiation, could increase surface MICA and MICB levels, ionizing radiation and MMP inhibitors were combined and used to treat NCI-H23 cells (Fig. 4). The inhibition of MMPs further enhanced the surface expression levels of MICA, which were induced by ionizing radiation. However, the relative surface 

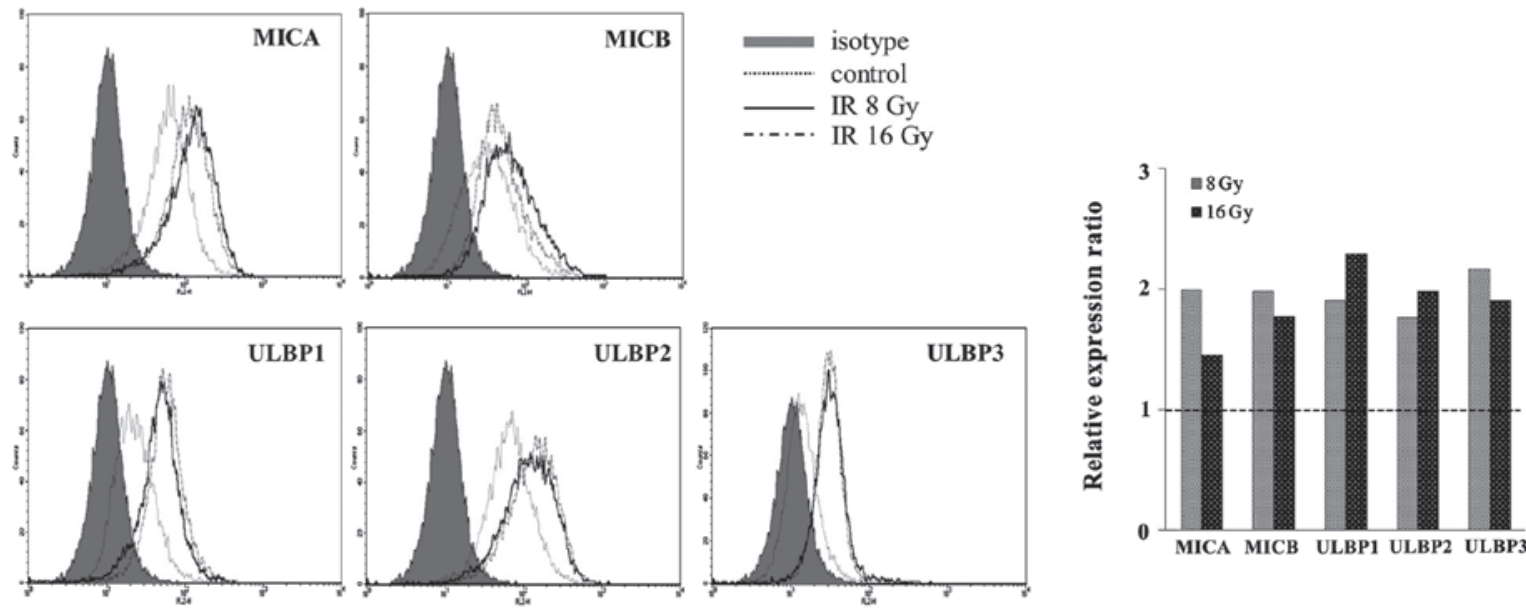

Figure 2. Increased surface expression levels of natural killer group 2 member D ligands (NKG2DLs) by ionizing radiation. NCI-H23 human non-small cell lung cancer cells were irradiated with the indicated doses. Following a $24 \mathrm{~h}$ recovery, the cells were harvested and assayed using flow cytometry, with five types of anti-NKG2DLs monoclonal antibodies. A high radiation dose could not further increase the surface major histocompatibility complex class-I polypeptide-related chain proteins (MIC)A and MICB levels. The surface expression of MICA and MICB in irradiated cells were demonstrated with histograms and the ratio of mean fluorescence intensities (MFI) compared with the control cells. The representative results are shown. IR, ionizing radiation; ULBP, UL-16 binding protein; Gy, gray unit.

A

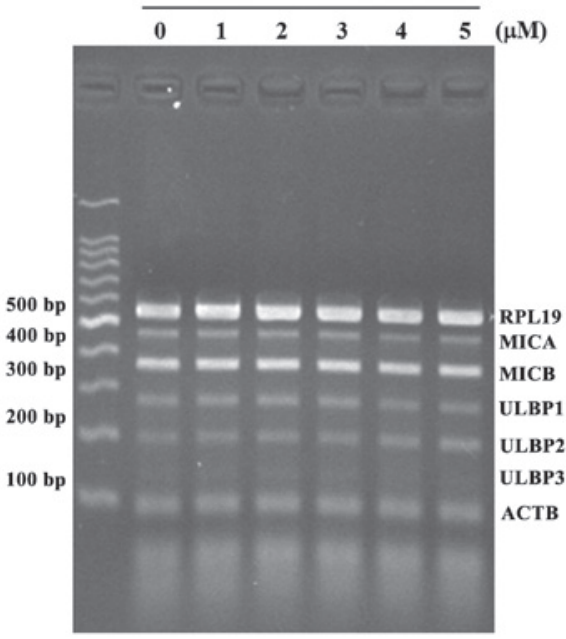

B

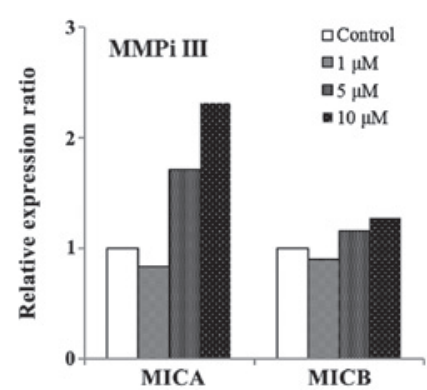

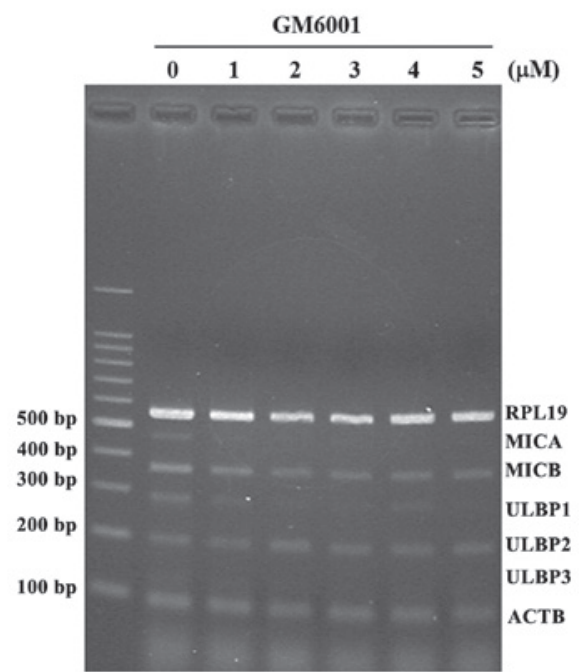

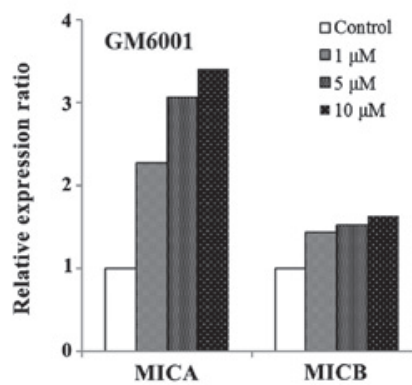

Figure 3. Increase in surface natural killer group 2 member D ligands (NKG2DLs) expression levels, without an increase in the mRNA expression levels, following treatment with matrix metalloproteinase (MMP) inhibitors. NCI-H23 human non-small cell lung cancer cells were treated with MMP inhibitor (MMPi) III or GM6001 at the indicated doses. Following an $18 \mathrm{~h}$ incubation, the cells were harvested and assayed using (A) quantitative polymerase chain reaction and (B) flow cytometry. The surface expression of MICA and MICB in MMP inhibitor treated cells were demonstrated the ratio of mean fluorescence intensities (MFI) compared to control cells. The representative results were showed. Bp, base pairs; ULBP, UL-16 binding proteins; MIC, major histocompatibility complex class-I polypeptide-related chain proteins; RPL19, ribosomal protein L19; ACTB, $\beta$-actin.

expression levels of MICB were not markedly altered by MMP inhibitors and irradiation co-treatment, as compared with irradiation treatment only. From these results, it was hypothesized that the preexisting MMPs may contribute the shedding of MICB and radiation induced MMPs only affected the shedding of MICA. 

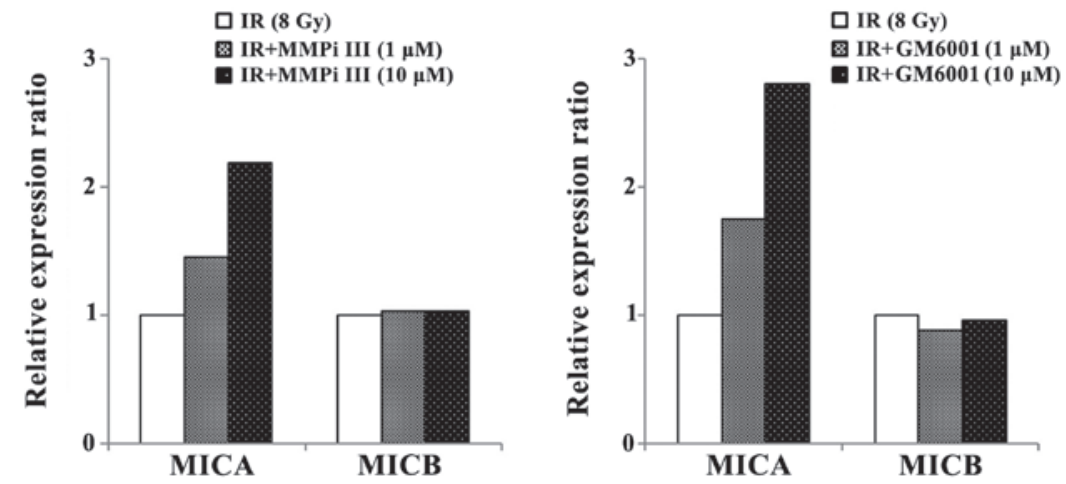

Figure 4. Increase in surface natural killer group 2 member D ligands (NKG2DLs) expression levels by treatment with matrix metalloproteinase (MMP) inhibitors following irradiation. NCI-H23 human non-small cell lung cancer cells were irradiated with 8 Gy and treated with MMPi III or GM6001 at the indicated doses, during recovery. Following an $18 \mathrm{~h}$ incubation, the cells were harvested and assayed using flow cytometry. Surface major histocompatibility complex class-I polypeptide-related chain protein (MIC)A was markedly increased in response to the combined treatment. The surface expression of MICA and MICB in combined treated cells were demonstrated the ratio of mean fluorescence intensities (MFI) compared to simply irradiated cells. The representative results were showed. IR, ionizing radiation; Gy, gray unit.
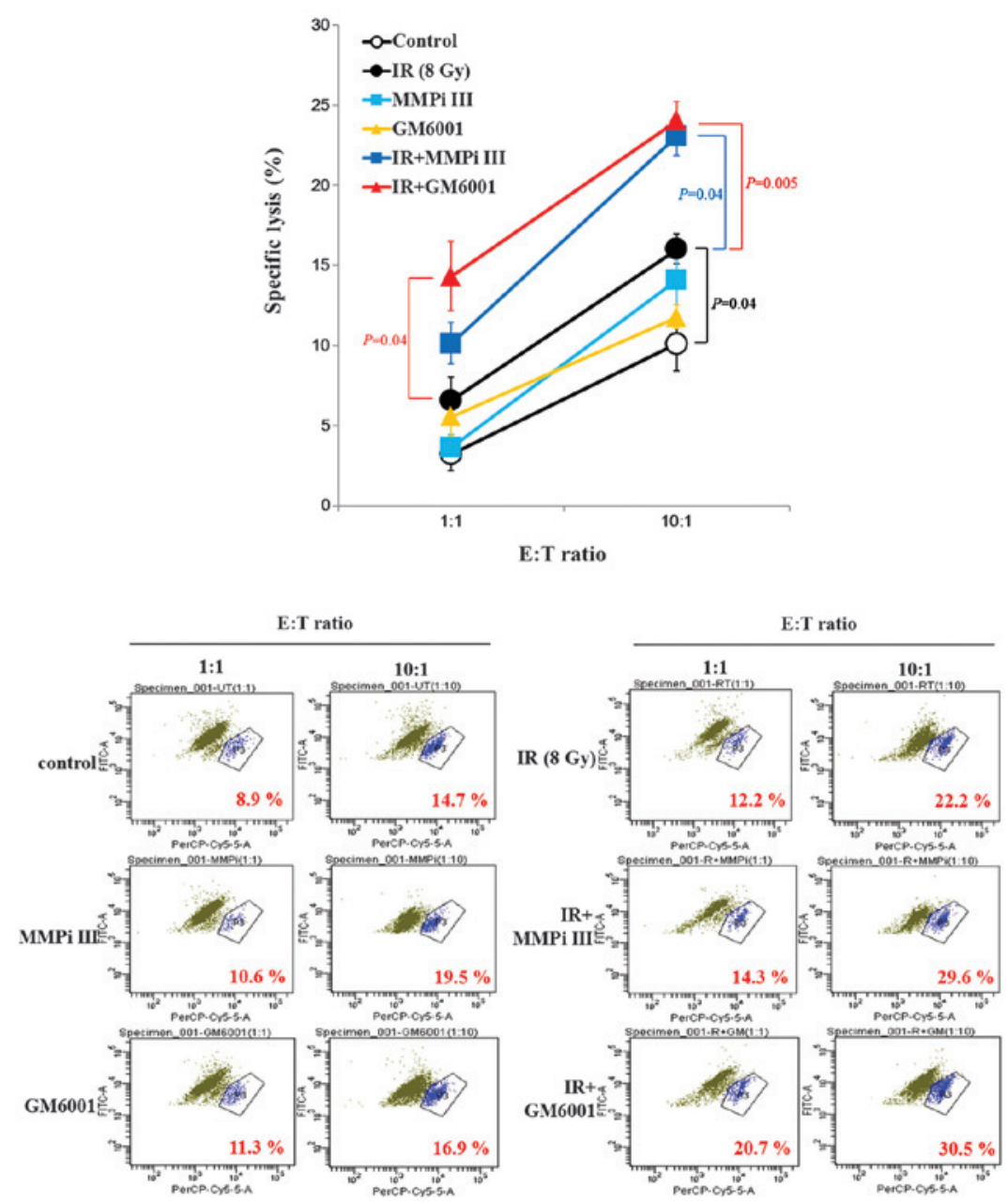

Figure 5. Enhanced susceptibility of NCI-H23 human non-small cell lung cancer cells to natural killer (NK) cells in response to treatment with matrix metalloproteinase (MMP) inhibitors, following irradiation. NCI-H23 cells were irradiated with 8 Gy and treated with MMP inhibitor (MMPi) III or GM6001 at the indicated doses, during recovery. Following an $18 \mathrm{~h}$ incubation, the cells were harvested and co-cultured with NK-92 natural killer cells. The cytotoxicity of the NK-92 cells was measured by the proportion of dead cells. Combined treatment markedly increased the cytotoxicity of NK-92 cells. IR, ionizing radiation; E:T, effector to target cell ratio; Gy, gray unit.

Susceptibility of NCI-H23 cells to NK cells is synergistically increased by the combination of ionizing radiation and MMP inhibitors. Consequently, it was determined whether the co-treatment of MMP inhibitors and irradiation, could increase the cytotoxic effects of NK cells on cancer cells. The MMP inhibitor treatment alone did not alter the cytotoxic NK 


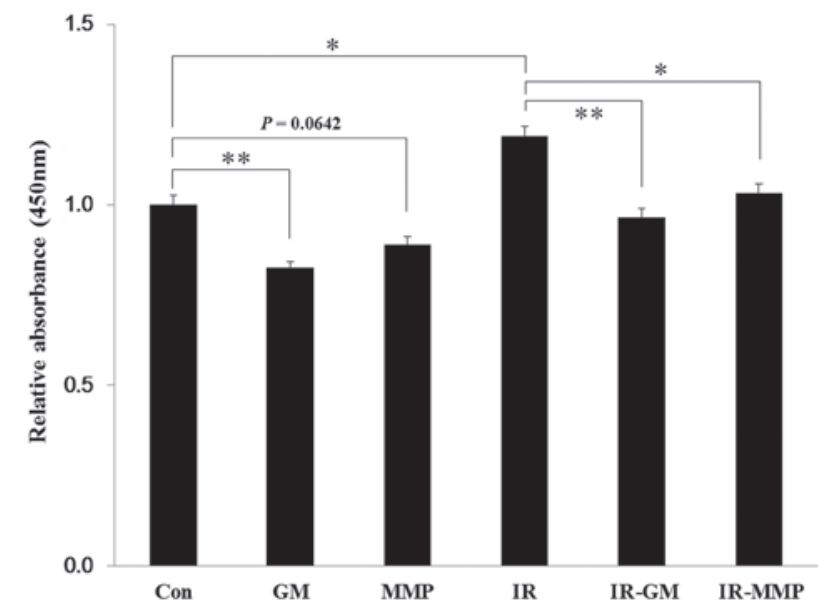

Figure 6. Reduction of soluble major histocompatibility complex class-I polypeptide-related chain protein (MIC)A by treatment with matrix metalloproteinase (MMP) inhibitors. The supernatants were collected from NCI-H23 human non-small cell lung cancer cells which were irradiated with 8 Gy and treated with MMP inhibitors. Soluble MICA was measured using an anti-MICA coated ELISA kit. " $\mathrm{P}<0.05,{ }^{* * *} \mathrm{P}<0.01$. Con, control; nm, nanometers; IR, ionizing radiation; Gy, gray unit.

effects and ionizing radiation treatment alone only minimally increased the cytolysis of cancer cells. However, the combined treatment synergistically increased the cytotoxicity of NK cells to NCI-H23 cells (Fig. 5). It remains unclear as to why the induction of surface expression levels of MICA and MICB, through the inhibition of MMPs, could not alter the cytotoxicity of NK cells.

Soluble NKG2D levels are increased by ionizing radiation, but blocked by MMP inhibitors. It was hypothesized that the increased levels of surface MICA, by treatment with MMP inhibitors, was due to the reduction in the shedding of soluble MICA. The levels of soluble MICA in the supernatants, which were collected from irradiated or MMP inhibitor-treated NCI-H23 cells, were quantified by ELISA. Treatment with the MMP inhibitors decreased the levels of soluble MICA in both the non-irradiated and irradiated samples, and ionizing radiation treatment alone increased the soluble MICA levels, as compared with the control (Fig. 6).

\section{Discussion}

NK cells exert cytotoxic effects on numerous cancer cells; however, anti-cancer immunity is generally ineffective against established cancer cells. Cytotoxicity of NK cells needs to be potentiated (17) and is controlled by the balance of signaling between stimulatory and inhibitory receptors. Therefore, the induction of activating ligands, such as NKG2DLs, is a potential approach to reactivate NK cells and increase cytolysis of cancer cells (18). The precise mechanisms of NKG2DL regulation however, remains unclear. It has previously been observed that there are discordances between the mRNA and surface protein expression levels of NKG2DLs, in response to numerous cell stresses $(3,19)$. Furthermore, the surface protein levels of NKG2DLs were increased, without alteration of mRNA expression, by ionizing radiation in A549 lung cancer cells (20). These results suggest that the expression levels of NKG2DLs are strictly regulated at the transcriptional level, as well as the post-transcriptional level. Prior to the present experiments, ionizing radiation was identified as being capable of markedly increasing the surface NKG2DLs levels, this may be through the ATM-ATR pathway at the post-transcriptional level, despite minimal alterations to the mRNA expression levels, in radioresistant cells (20). However, despite the observed significant induction of NKG2DLs, the enhancement of the susceptibility of the cancer cells to NK cells was minimal (3). This may be due to the increased expression levels of soluble NKG2DLs, MHC molecules and heat shock proteins (HSPs). The downregulation of MHC molecules and HSPs in previous studies, through the use of histone deacetylase inhibitors and heat shock factor inhibitors, respectively, have been shown to further enhance NK cell-mediated anticancer immunity $(20,21)$. Soluble NKG2DLs are shed through the proteolysis of extramembrane domains in membrane bound-proteins by numerous proteases including MMPs and ADAMs, or directly secreted by exosomes (22). The secreted and soluble NKG2DLs bind to NKG2D receptors on NKG2D+ immune cells, including NK cells, and downregulate the surface expression levels of NKG2D receptors (23). Eventually, this results in the lack of response of the immune cells to NKG2DLs+ cancer cells. The reduction of soluble NKG2DLs through the inhibition of MMPs may be an effective strategy to minimize the adverse effects of radiotherapy.

The present study aimed to determine whether ionizing radiation, a potent $\mathrm{NKG} 2 \mathrm{DL}$-inducer, may effectively enhance the susceptibility of cancer cells to NK cells, with the assistance of MMP inhibitors, through the reduction of soluble NKG2DLs. Although the mRNA expression levels of NKG2DLs were not increased by treatment with two MMP inhibitors, and MMP2 was upregulated by irradiation in NCI-H23 cells, the surface NKG2DLs levels synergistically increased, in response to the co-treatment of ionizing radiation and MMP inhibitors. Simultaneously, the concentration of soluble NKG2DLs, sMICA and SMICB, were increased by irradiation but reduced in response to MMP inhibition. Therefore, the combined effects of treatment of cancer cells with ionizing radiation and MMP inhibitors, may increase the cytotoxicity of NK cells.

Ionizing radiation efficiently induced the surface expression levels of NKG2DLs; however, it also increased the soluble expression levels of NKG2DLs and suppressed NK activity. Furthermore, MMPs have a role in cancer invasion and metastasis, and are associated with a poor prognosis in patients with cancer. Therefore, when radiotherapy is used in the treatment of patients with cancer, it may be beneficial to also block the activity of MMPs. The present study showed that MMP inhibition may further enhance the efficacy of NK cell-based anticancer immunotherapy, when combined with radiotherapy.

\section{Acknowledgements}

The present study was supported by a grant from the Pusan National University (2011-2012). Support was also provided by the National R\&D Program, through the Dongnam Institute of Radiological \& Medical Sciences, funded by the Ministry of Science, ICT and Future Planning (no. 50590-2014). Support 
was also provided by the National Research and Development Program for Cancer Control, Ministry for Health and Welfar, Republic of Korea (no. 0920050).

\section{References}

1. Friedman EJ: Immune modulation by ionizing radiation and its implications for cancer immunotherapy. Curr Pharm Des 8: 1765-1780, 2002.

2. Nakagawa K, Yoshida F, Omori N, Tsunoda T and Nose T: The effect of radiation therapy combined with natural killer cells against spontaneous murine fibrosarcoma. Biotherapy 2: 69-75, 1990.

3. Kim JY, Son YO, Park SW, et al: Increase of NKG2D ligands and sensitivity to NK cell-mediated cytotoxicity of tumor cells by heat shock and ionizing radiation. Exp Mol Med 38: 474-484, 2006.

4. von Essen CF: Radiation enhancement of metastasis: a review. Clin Exp Metastasis 9: 77-104, 1991

5. Wang CJ, Chen HC, Huang EY and Lee SP: Elective neck irradiation for nasopharyngeal carcinoma. Chang Gung Med J 23: 387-395, 2000.

6. Davidson B, Goldberg I, Kopolovic J, et al: MMP-2 and TIMP-2 expression correlates with poor prognosis in cervical carcinoma - a clinicopathologic study using immunohistochemistry and mRNA in situ hybridization. Gynecol Oncol 73: 372-382, 1999.

7. Ekinci T, Ozbay PO, Yiğit S, Yavuzcan A, Uysal S and Soylu F: The correlation between immunohistochemical expression of MMP-2 and the prognosis of epithelial ovarian cancer. Ginekol Pol 85: 121-130, 2014

8. Liu WW, Zeng ZY, Wu QL, Hou JH and Chen YY: Overexpression of MMP-2 in laryngeal squamous cell carcinoma: a potential indicator for poor prognosis. Otolaryngol Head Neck Surg 132: 395-400, 2005.

9. Boutet P, Agüera-González S, Atkinson S, et al: Cutting edge: the metalloproteinase ADAM17/TNF-alpha-converting enzyme regulates proteolytic shedding of the MHC class I-related chain B protein. J Immunol 182: 49-53, 2009.

10. Kohga K, Takehara T, Tatsumi T, et al: Anticancer chemotherapy inhibits MHC class I-related chain a ectodomain shedding by downregulating ADAM10 expression in hepatocellular carcinoma. Cancer Res 69: 8050-8057, 2009.
11. Lee BK, Kim MJ, Jang HS, et al: A high concentration of MMP-2/gelatinase A and MMP-9/gelatinase B reduce NK cell-mediated cytotoxicity against an oral squamous cell carcinoma cell line. In Vivo 22: 593-597, 2008.

12. Liu G, Atteridge CL, Wang X, Lundgren AD and Wu JD: The membrane type matrix metalloproteinase MMP14 mediates constitutive shedding of MHC class I chain-related molecule A independent of A disintegrin and metalloproteinases. J Immunol 184: 3346-3350, 2010

13. Sun D, Wang X, Zhang H, Deng L and Zhang Y: MMP9 mediates MICA shedding in human osteosarcomas. Cell Biol Int 35: 569-574, 2011.

14. Park SW, Bae JH, Kim SD, et al: Comparison of level of NKG2D ligands between normal and tumor tissue using multiplex RT-PCR. Cancer Invest 25: 299-307, 2007.

15. Eissmann P, Evans JH, Mehrabi M, Rose EL, Nedvetzki S and Davis DM: Multiple mechanisms downstream of TLR-4 stimulation allow expression of NKG2D ligands to facilitate macrophage/NK cell crosstalk. J Immunol 184: 6901-6909, 2010.

16. Gasser S, Orsulic S, Brown EJ and Raulet DH: The DNA damage pathway regulates innate immune system ligands of the NKG2D receptor. Nature 436: 1186-1190, 2005.

17. Lanier LL: NK cell recognition. Annu Rev Immunol 23: 225-274, 2005.

18. Ljunggren HG and Malmberg KJ: Prospects for the use of NK cells in immunotherapy of human cancer. Nat Rev Immunol 7: 329-339, 2007.

19. Bae JH, Kim JY, Kim MJ, et al: Quercetin enhances susceptibility to NK cell-mediated lysis of tumor cells through induction of NKG2D ligands and suppression of HSP70. J Immunother 33: 391-401, 2010.

20. Son CH, Keum JH, Yang K, et al: Synergistic enhancement of NK cell-mediated cytotoxicity by combination of histone deacetylase inhibitor and ionizing radiation. Radiat Oncol 9: 49, 2014

21. Kim JY, Bae JH, Lee SH, et al: Induction of NKG2D ligands and subsequent enhancement of NK cell-mediated lysis of cancer cells by arsenic trioxide. J Immunother 31: 475-486, 2008.

22. Chitadze G, Bhat J, Lettau M, Janssen O and Kabelitz D: Generation of soluble NKG2D ligands: proteolytic cleavage, exosome secretion and functional implications. Scand J Immunol 78: 120-129, 2013.

23. Groh V, Wu J, Yee C and Spies T: Tumour-derived soluble MIC ligands impair expression of NKG2D and T-cell activation. Nature 419: 734-738, 2002. 